\title{
DE LA RECONNAISSANCE À LA MISE EN FORME RITUELLE DES UNIONS DE MÊME SEXE EN FRANCE. ENQUÊTE AUPRĖS DE CATHOLIQUES GAYS ET LESBIENNES
}

\section{Del reconocimiento a la ritualización de las uniones de parejas del mismo sexo en Francia. Estudio sobre gays y lesbianas católicos}

\author{
Céline Béraud* \\ * Université de Caen-Normandie, Institut Universitaire de France \\ celine.beraud@gmail.com
}

\section{Mots-clés}

Controverse Mariage de même sexe

Catholicisme français Rituels

Palabras clave

Controversia

Matrimonio

homosexual

Catolicismo francés

Rituales

\begin{abstract}
Resumé
En 2012-2013, les débats relatifs à l'ouverture du mariage et de l'adoption aux couples de même sexe ont fait l'objet d'une importante controverse en France, controverse bien plus intense que celle qu'a connu l'Espagne sept ans plus tôt sur le même sujet. Parmi les opposants à la loi, les catholiques sont apparus comme des acteurs clés. Malgré un affichage non confessionnel, la condamnation traditionnelle de l'homosexualité par le magistère catholique s'est alors trouvée réaffirmée: les pratiques homosexuelles sont qualifiées d' "intrinsèquement désordonnées" et condamnées car ne respectant pas "l'ordre de la nature". La mobilisation a constitué une épreuve pour les catholiques gays et lesbiens très intégrés à l'Eglise, auprès de qui j'ai mené une enquête qualitative sociologique. L'enquête montre cependant qu'ils et elles parviennent à faire cohabiter ces deux dimensions de leur identité. Ils obtiennent même un accompagnement voire une mise en forme rituelle de leur union par un prêtre.
\end{abstract}

\section{Resumen}

Entre los años 2012 y 2013, los debates en torno al derecho al matrimonio y a la adopción para parejas del mismo sexo han estado revestidos de una importante controversia en Francia, controversia mucho más intensa que en España siete años antes en relación al mismo tema. Entre los opositores a la ley, los católicos aparecen como actores clave. A pesar de los discursos no confesionales, la condena tradicional de la homosexualidad por parte del magisterio católico se ha visto reforzada: las prácticas homosexuales son calificadas de "desórdenes intrínsecos" y condenadas por no respetar "el orden de la naturaleza". La movilización ha constituido un reto para los católicos gays y lesbianas altamente integrados en la Iglesia; grupo sobre el que he realizado una investigación sociológica cualitativa. La investigación muestra que ellos y ellas consiguen, no obstante, hacer cohabitar estas dos dimensiones de su identidad obteniendo, incluso, un acompañamiento a través de una ritualización de su unión por parte de un cura.

Béraud, C. (2016). De la reconnaissance à la mise en forme rituelle des unions de même sexe en France. Enquête auprès de catholiques gays et lesbiennes. Papeles del CEIC, vol. 2016/2, papel 158, CEIC (Centro de Estudios sobre la Identidad Colectiva), Universidad del País Vasco, http://dx.doi.org/10.1387/pceic.16294 
Les travaux sociologiques sur l'homosexualité se sont développés en France depuis les années 1990, en lien d'abord avec le Sida, puis la conjugalité et la parentalité ${ }^{\prime}$. Si les thématiques se sont diversifiées, les recherches qui ont porté sur la religion sont encore peu nombreuses. Dans l'ouvrage issu de sa thèse, Hélène Buisson-Fenet (2004) s'est intéressée, en s'appuyant sur le cas français, aux modalités d'énonciation par l'Église catholique de l'interdit des relations homosexuelles, position sur laquelle elle s'arc-boute alors que la stigmatisation des personnes gays et lesbiennes a fortement décru et que la reconnaissance juridique de leurs unions a progressé au cours de la période étudiée, du début des années 1970 à la fin des années 1990. Loin de se limiter à l'étude de discours, l'auteure a analysé les différentes modalités d'appropriation de la norme par les acteurs catholiques. Elle a ainsi montré comment, sur le terrain ecclésial, les clercs s'attachent à "résoudre la dissonance entre une homosexualité condamnée par le magistère et une homosexualité qu'il faut bien accompagner" (BuissonFenet, 2004: 156). La dissonance est encore plus forte pour les prêtres et religieux homosexuels exposés, pas tant à la coercition canonique, qu'à un contrôle social diffus. Ils élaborent des accommodements avec l'institution dont les représentants feignent l'ignorance tant que les apparences sont sauvegardées. Mis à part cet important travail d'Hélène Buisson-Fenet, Martine Gross, spécialiste de I'homoparentalité, est alors la seule sociologue à s'intéresser aux rapports entre homosexualité et religion en France ${ }^{2}$, en abordant le judaïsme et le christianisme (Gross, 2004, 2008 et 2012). Il s'agit pour elle, en particulier, de dégager les stratégies de conciliations entre des identités a priori difficilement conciliables: celle d'homosexuel-le et celle de croyant se reconnaissant une appartenance à une tradition religieuse qui condamne les pratiques homosexuelles.

Dans le sillage de ces travaux, je me propose ici d'aborder la question des liens entre catholicisme et union de même sexe, non pas à travers l'étude des mobilisations conservatrices qu'a suscité en France comme dans d'autres pays l'ouverture du mariage à ces couples, mais en se situant à l'échelle des individus gays et lesbiens qui se revendiquent

\footnotetext{
${ }^{1}$ Voir le bilan de la littérature de Rault (2016).

${ }^{2}$ On doit également citer la thèse de Baptiste Coulmont (2003). Mais celle-ci porte sur les rituels de mariage de même sexe dans des Eglises protestantes américaines.
} 
catholiques pratiquant-e-s et entendent non seulement avoir recours aux dispositifs civils de reconnaissance de leur couple, mais parviennent aussi à négocier auprès d'un prêtre une mise en forme rituelle. Pour cela, j'ai recours à des travaux de sociologie des religions et aussi à ceux relevant d'une sociologie du mariage. Ces derniers pointent une désaffection croissante des couples à l'égard du mariage en France depuis 1972 (Bozon, 1992; Segalen, 2003). A l'exception d'un sursaut au début des années 2000, la tendance à la baisse n'a pas été inversée. On est ainsi passé de 417.000 mariages célébrés en 1972 à 239.000 en 2015. Cette tendance n'a pas empêché les couples qui y ont encore recours d'investir fortement, tant d'un point de vue financier que symbolique, les cérémonies sur le mode de la personnalisation de la fête, sans échapper d'ailleurs pour autant à une certaine standardisation des pratiques (Maillochon, 2016). Les travaux récents qui sont consacrés à ce phénomène (Maillochon, 2014; 2016) ignorent très largement la dimension religieuse des cérémonies. Or, en 2012, sur les 245.930 mariages civils célébrés, 70.369 ont donné lieu à une mise en forme rituelle catholique, soit $28,5 \%$ (contre un tiers en $2005^{3}$ ). Si la part des mariages religieux a fortement décliné, elle est donc loin d'être négligeable. Surtout, le catholicisme offre un patrimoine architectural et liturgique inégalé en France pour satisfaire les attentes de couples en quête de solennisation et d'esthétisation de leur union.

Après avoir rappelé les deux grandes étapes de l'institutionnalisation des couples de même sexe en France, qui ont constitué deux moments de mise à l'épreuve de mes enquêté-e-s du fait des résistances catholiques qui se sont alors exprimées, on s'intéressera à leurs pratiques matrimoniales et à leur remarquable capacité à créer des événements conjugaux, civils et religieux.

\section{Présentation de l'enquête}

Cette enquête trouve son origine dans un travail d'analyse de la controverse relative au mariage pour tous réalisé avec Philippe Portier dans le cadre d'une recherche européenne dirigée par Alfonso Pérez-Agote sur les "nouveaux" conflits entre religion et politique dans des pays de tradition catholique du Sud de l'Europe (Belgique, Espagne, France, Italie et Portugal) (Dobbelaere et

\footnotetext{
${ }^{3}$ Au cours de la décennie passée, la baisse du nombre de mariages catholiques a été plus importante que celle du nombre de mariages civils.
} 
Pérez-Agote, 2015). Après ce premier travail (Béraud et Portier, 2015), j'ai souhaité étudier les catholiques qui étaient favorables à la nouvelle loi (Béraud, 2015) et plus précisément, celles et ceux qui entendaient y avoir personnellement recours et qui étaient très largement restés dans l'ombre, suscitant peu d'intérêt dans les médias et encore moins dans le champ académique.

Ainsi, cet article s'appuie sur le matériau recueilli à l'occasion d'une enquête de terrain, encore en cours, auprès de catholiques pratiquant-e-s réguliers, en couple de même sexe, qui ont fait le choix de se marier ou ont pour projet de le faire prochainement. Comment ces catholiques investissent-ils le mariage civil, dont les autorités religieuses ont pourtant proclamé qu'il ne pouvait unir qu'un homme et une femme, au nom du principe de différence des sexes qu'elles jugent irréductible? Cette première question a très vite été complétée par une seconde, dont l'ampleur est apparue dès les premiers entretiens réalisés au printemps 2014. Comment l'institution ecclésiale et ses agents, les prêtres en particulier, répondent-ils, souvent dans la plus grande discrétion, aux demandes d'accompagnement voire de mise en forme rituelle de leur union qui émanent de fidèles gays et lesbiennes? J'ai pour l'instant réalisé une dizaine de longs entretiens biographiques au domicile des personnes afin de saisir leurs trajectoires religieuse et conjugale, entretiens que je souhaiterais compléter par des entretiens avec des prêtres qui accompagnent ces fidèles, voire des observations des rituels accomplis.

$\mathrm{Si}$ "se revendiquer homosexuel et fervent catholique semble une combinaison improbable" (Gross, 2012: 215), être catholique pratiquant et accéder au mariage avec une personne du même sexe l'est encore davantage. II s'agit d'un sujet "tête d'épingle": les catholiques pratiquants réguliers constituent aujourd'hui une minorité; ceux qui parmi eux vivent en couple de même sexe et de surcroît mariés représentent une minorité au sein de cette minorité. II n'est donc pas simple de constituer un échantillon. Souhaitant dans un premier temps rencontrer des fidèles "ordinaires" (c'est-à-dire intégrés aux structures ordinaires catholiques, principalement paroissiales), j'ai fait le choix de ne pas m'adresser d'abord aux réseaux catholiques gay friendly. J'ai procédé par effet "boule de neige" demandant aux personnes rencontrées de me mettre en contact avec d'autres couples. De ce fait, et il s'agit là d'un biais, mes enquêté-e-s partagent certaines caractéristiques sociales, notamment le fait d'appartenir aux classes supérieures de la région parisienne et d'être pour la plupart des trentenaires et des quadragénaires. Touchant doublement à l'intime de la foi et de la sexualité, plusieurs de mes demandes sont restées 
sans réponse. Celles et ceux qui y ont répondu ont déjà témoigné publiquement: dans des groupes catholiques, voire dans les médias lors de l'épisode du mariage pour tous. Ils parviennent à concilier deux dimensions a priori contradictoires de leur identité: leur appartenance religieuse et la conjugalité voire la parentalité qui est la leur et que le magistère catholique désapprouve.

\section{ReConNaissance des COUPLES DE MÊME SEXE ET RÉSISTANCES CATHOLIQUES}

On a pu parler d'une "surmoralisation" du modèle bioéthique français (Pulman, 2010: 295). De la même façon, on peut évoquer en France une sécularisation plus lente du mariage ${ }^{4}$, "institution [certes] sécularisée [mais] toujours empreinte de représentations et de connotations religieuses" (Rault et Retrait, 2009: 43). Ainsi, le mariage s'ouvre, avec la loi du 18 mai 2013, aux couples de même sexe plus tardivement et de manière plus conflictuelle que dans d'autre pays européens pourtant de tradition catholique majoritaire: Belgique (2003), Espagne (2005) et Portugal (2010).

\subsection{Le Pacs en 1999}

Différents projets visant à une institutionnalisation des unions de même sexe se font jour au cours des années 1990. Ils se concrétisent en 1999 avec le Pacs (Pacte civil de solidarité) qui va pendant quatorze ans constituer "la solution française à la reconnaissance légale du couple de même sexe" (Rault, 2008: 117). Celle-ci se distingue des autres dispositifs européens par deux aspects: les couples de sexes différents peuvent aussi y avoir recours; le statut offert est assez différent de celui du mariage civil.

La proposition de loi débattue au Parlement en 1998-1999, suscite à son encontre une importante mobilisation catholique. Ainsi, le 17 septembre 1998, le Conseil permanent de l'épiscopat publie un communiqué au titre explicite: "Le Pacte civil de solidarité (PACS): 'Une loi inutile et dangereuse'". II y est question d'anthropologie, de symbolique et de différence des sexes. En revanche, on ne trouve pas dans ce texte de références faites à la Bible, aux Pères de l'Eglise ou au magistère romain (le Catéchisme de l'Eglise catholique). "Un athée aurait pu le signer" s'en

\footnotetext{
${ }^{4}$ Sur ce point voir Portier, Théry, Béraud et Coulmont (2015). 
amuse Eric Fassin (2001: 99). La déclaration de l'épiscopat s'adresse à la "société toute entière" et non pas seulement aux catholiques. Si I'Eglise s'investit intensément dans le débat d'opinion, elle intervient également “(...) par l'influence directe qu'elle exerce sur la position des hommes politiques" (Buisson-Fenet, 2004: 21). Ainsi, la droite fait très largement chœur avec l'Eglise. Le combat anti-Pacs donne lieu à plusieurs mobilisations et manifestations portées par des troupes très largement catholiques, dont plusieurs éléments se rejoueront lors des débats sur le "mariage pour tous".

Si les couples gays et lesbiens en ont été chronologiquement les tout premiers usagers, le Pacs rencontre très vite un succès croissant auprès de couples hétérosexuels qui souhaitent inscrire leur union dans un cadre juridique sans pour autant avoir recours au mariage, ar rapport auquel il offre désormais une véritable alternative. Depuis 2010, ces derniers représentent chaque année $95 \%$ des contractants. Au cours de la même période, on compte deux Pacs hétérosexuels conclus pour trois mariages célébrés (Bellamy et Beaumel, 2014).

Le Pacs (retouché en 2005, 2006, 2007 et 2011 pour le rapprocher de celui des couples mariés sans le rendre pour autant équivalent) conduit juridiquement à une transformation réelle de la vie commune des couples non mariés, au plan du droit des biens, de la propriété, du droit de la sécurité sociale et de l'imposition. Il établit aussi des devoirs réciproques aux contractants (en matière d'assistance matérielle), sans toucher cependant à la question de l'adoption non plus qu'à celle de l'autorité parentale ou à la procréation médicalement assistée. En outre, il n'est en principe pas conclu en mairie avec la solennité du mariage, mais au tribunal ou devant notaire (depuis 2012). Des militants et des intellectuels y ont vu d'une "union au rabais" ${ }^{5}$ pour les couples de même sexe. Daniel Borrillo et Pierre Lascoumes (2002) ont quant à eux décrit "une controverse inachevée".

\subsection{L'épisode du "mariage pour tous", 2012-2013}

L'Espagne a ouvert en 2005, c'est-à-dire huit ans avant la France, le mariage aux couples de même sexe (Pichardo Galán, 2008). Si I'Eglise catholique a constitué la principale force de résistance, on n'a observé dans ce pays rien de comparable à la mobilisation française contre le

\footnotetext{
${ }^{5}$ Libération, 15 mars 1999. 
"mariage pour tous" en 2012-2013, dont on a montré que le principal moteur était le catholicisme malgré un affichage non confessionnel (Béraud et Portier, 2015). En France, la question de la filiation a été centrale et fortement chargée d'un point de vue symbolique, alors qu'elle est apparue ailleurs (en Belgique et en Espagne) surtout dans ses dimensions strictement juridiques et traitée de manière "technique" (Paternotte, 2011: 38).

L'ouverture du mariage et de l'adoption aux couples de même sexe faisait partie des engagements de campagne de François Hollande, élu Président de la République française le 6 mai 2012. Le gouvernement annonce la mise en œuvre de son projet par la voix de la Ministre de la justice, Christiane Taubira, le 11 septembre 2012. Le débat va se dérouler sur plusieurs mois. La loi est définitivement votée le 23 avril 2013 par l'Assemblée nationale, et promulguée par le Président, après que le Conseil constitutionnel eut souligné sa conformité à la Constitution, le 18 mai 2013.

Pendant toute la période du débat parlementaire, les évêques multiplient les interventions: en interpellant d'abord le Président de la République et son gouvernement puis en appelant les parlementaires ainsi que les fidèles à se mobiliser. Ils n'ont d'ailleurs pas attendu 2012 pour le faire. Depuis le milieu des années 2000, ils s'attachent en effet, en visant l'opinion publique, à jouer le rôle de "lanceurs d'alertes" (Chateauraynaud et Torny, 1999) pour dénoncer la grave menace qui pèse selon eux sur la famille et la filiation. L'épiscopat suit ainsi de près la ligne défendue par le Saint-Siège, notamment dans la déclaration de la Congrégation pour la Doctrine de la Foi (2003), selon laquelle "il n'y a aucun fondement pour assimiler ou établir des analogies, même lointaines, entre les unions homosexuelles et le dessein de Dieu sur le mariage et la famille".

Si la contestation du projet de loi est de manière très nette le fait des évêques, les fidèles interviennent aussi, massivement, dans ce combat. On aurait tort d'y voir de simples coopérateurs de la hiérarchie. On se situe bien davantage, en raison de la subjectivation des comportements qui affecte aussi le monde catholique, y compris du côté de sa fraction la plus identitaire, dans un mécanisme circulaire, qui voit les deux polarités se conforter mutuellement. Le mouvement s'agrège début septembre 2012 autour d'un collectif, qui prendra au cours de l'automne l'appellation "La Manif pour tous", en réponse euphonique à l'expression 
"mariage pour tous" qui est celle du gouvernement. Malgré un affichage non confessionnel, le poids des associations catholiques (associations familiales catholiques, groupements relevant de la mouvance "pro-vie" notamment) est au sein du collectif très important. A sept occasions en 2012-2014, les foules de la "Manif pour tous", fières d'elles-mêmes, ont défilé, persuadées en défendant l'hétéronormativité de lutter en faveur du "bien commun", au nom du respect de l'ordre "naturel" des corps voulu par Dieu voire de la pseudo évidence du biologique (celle de la différence anatomique des sexes) et de la force de conviction du "bon sens" comme l'ont proclamé certains slogans.

En s'inscrivant dans la perspective qui est celle du droit naturel et en empruntant des termes au lexique des sciences humaines -comme l'invocation de "fondements anthropologiques" qui avaient joué le rôle de référence voire de "transcendance" de substitution lors des débats sur le Pacs (Fassin, 2001: 95)-, les anti mariage pour tous, clercs ou militants laïcs, ont entendu opérer une montée en généralité voire prétendre à l'universalité des normes défendues. Pour mener à bien ce combat moral, ils ont noué en externe des alliances religieuses (avec les représentants d'autres cultes) (Béraud, 2014) et non-religieuses (principalement du côté de certains "psy" et certains juristes) afin de désingulariser leur cause et en effacer la dimension confessionnelle. En interne, ils ont du préserver le consensus, ce qui les a conduits à construire et conserver cette façade institutionnelle d'unanimité.

\subsection{Mises à l'épreuve}

Mes enquêté-e-s connaissent parfaitement le corpus contemporain de textes romains qui qualifient les "actes d'homosexualité" $d^{\prime \prime \prime}$ intrinsèquement désordonnés" et les condamnent car ne respectant pas "I'ordre de la nature" ${ }^{6}$. D'un point d'un point de vue pastoral, ces mêmes textes prônent l'accueil et le soutien des homosexuels "dans l'espoir de surmonter leurs difficultés personnelles et leur inadaptation

\footnotetext{
${ }^{6}$ Déclaration sur certaines questions d'éthique sexuelle (Persona Humana, Congrégation pour la doctrine de la Foi, 29 décembre 1975); Orientations éducatives sur l'amour humain (Instruction de la Congrégation pour l'éducation catholique, 1983); Lettre aux évêques de I'Eglise catholique sur la pastorale à l'égard des personnes homosexuelles (Congrégation pour la doctrine de la foi, 30 octobre 1986); Observations au sujet des propositions de loi sur la non-discrimination des personnes homosexuelles (Congrégation pour la doctrine de la foi, 1992). Pour une analyse sociologique de ce corpus, voir Buisson-Fenet (2004: 57-64).
} 
sociale" ${ }^{7}$. Malgré ce principe affiché d'accueil des personnes, les catholiques que j'ai rencontrés, qui refusent de "compartimenter" leur vie religieuse et leur vie en couple de même sexe ${ }^{8}$, ont tous et toutes connu des expériences négatives en paroisse. Pour autant, la plupart n'a pas souhaité se tourner vers des espaces ecclésiaux gay friendly, qui permettent des formes de communalisation religieuse sans risque $e^{9}$. Et ceux qui l'ont fait n'ont pas pour autant abandonné leurs pratiques paroissiales ordinaires.

Comme lors des débats relatifs à l'adoption du Pacs, l'épisode du mariage pour tous a "réveillé une homophobie latente" dans les milieux catholiques (Besson, 2012: 12). II a constitué un moment paroxystique dans la mise à l'épreuve de mes enquêté-e-s ${ }^{10}$. Très engagés et très attachés à l'institution ecclésiale, ils ont été confrontés à la mobilisation des anti, non seulement dans l'espace public et médiatique mais aussi dans le cadre paroissial de leurs pratiques, l'épisode de la mobilisation contre le mariage pour tous ayant pris des formes liturgiques. Des curés n'ont pas hésité à appeler, en chaire, leurs paroissiens à interpeller leurs élus et à descendre dans la rue, renouant avec une forme de "politisation" de certaines "pratiques sacerdotales" qui ne sont pas sans évoquer celles décrites par Yves Déloye (2006) à propos de l'engagement d'ecclésiastiques sur le terrain électoral sous la llle République, pratiques tombées en désuétude depuis une bonne cinquantaine d'années.

Les propos recueillis auprès de mes enquêté-e-s témoignent du sentiment de marginalisation voire d'exclusion vécu par les catholiques pratiquant-e-s en couple de même sexe lors de la mobilisation contre le mariage pour tous, lancée par le président de la conférence épiscopale avec la prière du 15 août 2012 puis relayée dès l'automne avec les rassemblements de la Manif pour tous:

\footnotetext{
${ }^{7}$ Apparaît ici un autre topos du discours catholique contemporain sur I'homosexualité: celui d'un vécu considéré comme nécessairement douloureux.

${ }^{8} \mathrm{Ce}$ qui constitue une des stratégies dégagées par Martine Gross (2012) pour gérer la contradiction entre les identités en conflits. Stratégie qui va de pair avec une forme d'invisibilité de la vie de couple en paroisse. Elle consiste à "maintenir l'identité sexuelle de la personne dans des espaces et des temps différents de ceux de l'Église".

${ }^{9}$ Il s'agit là d'une autre stratégie dégagée par Martine Gross (2012: 221-223).

${ }^{10}$ Philippe Clanché (2014) décrit dans le chapitre 10 de son ouvrage "Le traumatisme des homos cathos", spécifiquement entre les pages 123 et 136.
} 
On a utilisé le 15 août la prière universelle ${ }^{11}$ comme un mot d'ordre de mobilisation politique. Moi, ça m'a fait vraiment très mal. C'était comme un coup de poignard dans le dos (...). Et après, en novembre, sont arrivées les manif (...). Dans notre paroisse, tous les dimanches, c'était soit dans la prière universelle, soit dans l'homélie, soit à la fin de l'office par un appel à manifester, à avoir des volontaires... il faut s'inscrire pour faire la sécurité de la manif... À la limite, c'était comme si à la fin de la messe on allait y ensemble. II y avait quelques personnes très mobilisées parmi les fidèles. Pendant six mois, ça a été vraiment très dur. Je sortais au milieu de la messe en pleurant, quand je rentrais à la maison Cédric me ramassait à la petite cuillère... (François, 34 ans, ingénieur, pratiquant régulier, en couple de même sexe depuis 15 ans).

Bertrand (45 ans, pratiquant régulier, en couple depuis 10 ans), qui se décrit comme "intimement blessé", relate l'appréhension qu'il ressentait alors en allant à la messe: "Je redoutais I'homélie, la prière universelle". II raconte s'être levé pour quitter l'église lorsqu'un appel à manifester a été lancé pendant la messe, à laquelle il assiste sur son lieu de vacances. Dans les deux paroisses qu'elles fréquentent alors alternativement, Jeanne et Bénédicte ( 40 ans, mariées, en couple depuis 20 ans) semblent avoir été moins exposées.

La mobilisation catholique contre le mariage pour tous a conduit certain-e-s de mes enquêté-e-s à des prises de paroles, d'autres à des formes de sorties temporaires et beaucoup à l'expression d'une loyauté malgré les souffrances endurées (Béraud, 2015). Leurs prises de parole ont pu trouvé des cadres collectifs. Contrairement à l'aspect très monolithique qu'a donné à voir la mobilisation catholique, celle-ci n'a en effet pas pour autant été unanime. Bien qu'assez largement étouffées par la hiérarchie et peu relayées par les médias, des voix dissonantes favorables au projet de loi se sont exprimées, du côté des catholiques contestataires sensibles aux questions de genre qui portent la cause des femmes et celles des personnes LGBT, mais également au-delà de ces

\footnotetext{
${ }^{11}$ Renouant avec la pratique de "prière pour la France" qui était tombée en désuétude depuis plusieurs décennies, le cardinal Vingt-Trois, alors président de la conférence des évêques de France, a recours au répertoire liturgique. II transmet aux diocèses un texte à lire dans les paroisses lors de la messe du 15 août 2012. Les fidèles s'y trouvent invités à prier pour que "les enfants et les jeunes (...) cessent d'être les objets des désirs et des conflits des adultes pour bénéficier pleinement de l'amour d'un père et d'une mère" mais également pour leurs élus afin que "leur sens du bien commun de la société l'emporte sur les requêtes particulières et qu'ils aient la force de suivre les indications de leur conscience".
} 
groupes militants (Béraud, 2015), donnant à voir la pluralité interne au catholicisme français.

\section{RituALISATIONS MATRIMONIALES DE CATHOLIQUES EN COUPLE DE MÊME SEXE}

Les catholiques pratiquant-e-s en couple de même sexe que j'ai rencontrés apparaissent particulièrement habiles pour créer, suivant l'expression de Florence Maillochon (2016), des "événements conjugaux" civils et religieux, jusqu'à me dire comme Nicolas qu'ils avaient l'impression de s'être "mariés plusieurs fois". Malgré l'opposition de leur Eglise à ces formes d'union, ils ont choisi de se pacser et ont profité du mariage de même sexe, dès que la loi l'a rendu possible. Chacun de ces moments a donné lieu à des cérémonies particulièrement soignées. Plus inattendu encore, ils ont obtenu un accompagnement et mise en forme rituelle de leur union par un prêtre.

\subsection{Du Pacs au mariage civil}

Mes enquêté-e-s, avant que ne s'ouvre à eux la possibilité de se marier, ont tous et toutes contracté un Pacs, ce qui peut paraître surprenant, les catholiques ayant moins tendance à se pacser que les sans-religion (Rault et Retrait, 2009). On peut estimer que c'est en raison d'une "matrimonialisation impossible" qu'ils et elles ont eu recours à ce dispositif et l'ont fortement ritualisé:

"Il s'agit de la situation où se trouvent celles et ceux qui veulent se marier et ne le peuvent pas. Faute de pouvoir accéder à l'institution matrimoniale, les couples de même sexe peuvent faire jouer au Pacs un rôle de mariage de substitution. Dès lors, les partenaires recherchent sa ritualisation grâce à l'emprunt de séquences typiquement 'matrimoniales': désignation de témoins, reproduction d'un échange de consentement, échange d'alliances, etc." (Rault, 2008: 118).

Leur union s'est trouvée mise en scène en ayant recours non seulement au répertoire républicain mais aussi au répertoire catholique (voir plus loin). Cette première reconnaissance civile de leur union n'a pas été vécue comme une simple formalité administrative mais construite comme un moment solennel et festif, lui conférant ainsi une forte 
dimension symbolique ${ }^{12}$. L'engagement conjugal qu'ils prennent à cette occasion est à la fois ritualisé et publicisé. Ainsi, Nicolas et Louis, lorsqu'il se pacsent en 2009 , font une "fête en mairie"13. Ils bénéficient alors de l'accueil particulièrement soigné d'un adjoint au maire qui les reçoit avant pour mieux les connai itre et discuter de leur engagement, "un peu comme un prêtre aurait pu le faire", explique Nicolas. Bertrand et Charles font de même en 2011. Ils organisent à cette occasion une "petite fête" avec les enfants de Charles (nés de son précédent mariage avec une femme) et quelques amis.

L'étape suivante que constitue le mariage, rendue possible par la loi du 18 mai 2013, apparaît comme une évidence à ces personnes. Ils font partie des premier-e-s marié-e-s en couple de même sexe ${ }^{14}$. Nicolas et Louis se marient dès juillet. C'est aussi le cas de Jeanne et Bénédicte qui constituent le premier couple de même sexe uni civilement dans la ville où elles résident. Charles et Bertrand s'unissent dans le petit village normand où ils possèdent une résidence secondaire. Ils sont le premier couple d'hommes à s'y marier: "On a notre photo dans la gazette du village", déclarent-ils fièrement.

Outre "l'évidence" que plusieurs évoquent, d'autres raisons sont aussi mentionnées. Il s'agit aussi pour Nicolas d'un "acte politique". Celui-ci considère comme Jeanne qu'il fallait profiter sans tarder de "ce droit". L'ampleur de la mobilisation des anti semble également avoir accéléré les choses: "En 2008, si l'on m'avez demandé si je voulais me marier en tant qu'homosexuel, je n'aurais peut-être pas dit oui. (...) Avec cette radicalisation, c'est devenu un acte évident pour nous", considère Charles. Bertrand, son époux, ajoute que "le déchainement" les a conduit non seulement "à faire vite" mais aussi à se marier "de façon la plus publique posible". Enfin, pour les couples de femmes avec enfant(s) conçu(s) par AMP (Assistance Médicale à la Procréation), le mariage

\footnotetext{
${ }^{12}$ Voir la typologie de W. Rault (2007).

${ }^{13}$ Dans certaines villes, les maires acceptent de célébrer des Pacs dans leur salle des mariages. La cérémonie fait alors suite à la conclusion du Pacs au tribunal d'instance. Elle est purement symbolique et ne revêt donc aucune valeur légale. Les Pacs en mairie, pratique jusqu'alors officieuse, sont appelés à se généraliser avec la loi du 24 mai 2016, qui transfère leur enregistrement et leur dissolution aux communes. Les couples peuvent aussi avoir recours à un notaire.

${ }^{14}$ On compte 7.367 mariages de personnes de même sexe en 2013 sur un total de 238.592 mariages (c'est-à-dire 3\% de l'ensemble), 10.522 en 2014 sur un total de 241.292 (4,4\%) et 8.000 en 2015 sur un total de 239.000 (3,4\%). Source: Institut National des Études Démographiques (INED).
} 
apparaît comme un préalable à l'adoption par la seconde mère. D'autres dimensions, moins pressantes mais fondamentales, sont aussi mis en avant: des considérations patrimoniales bien sûr mais aussi le fait qu'ils adhèrent aux valeurs républicaines qui y sont associées comme le souligne François:

Se marier civilement ça veut dire se promettre fidélité et assistance et on pense que la société française se portera mieux quand il y aura plus de monde qui se seront promis assistance et fidélité. Parce que finalement dire qu'on se promet assistance c'est décharger Marianne du souci de me venir en assistance si je suis dans la merde ou de venir en assistance à Cédric s'il est dans la merde et que c'est un cadeau qu'on fait à Marianne en se mariant, en disant écoute c'est bon, nous deux on se gère, quoi. D'une part et puis la fidélité ben c'est ce qu'on vit comme une réalité. (François, 34 ans, ingénieur, pratiquant régulier, en couple de même sexe depuis 15 ans).

Comme l'avait été leur Pacs, la cérémonie de leur mariage civil est particulièrement soignée, voire sophistiquée. Ainsi, Nicolas et Louis adoptent comme un pied de nez à la "Manif pour tous" et aux mobilisations catholiques anti-gender, le rose et le bleu comme dress$\operatorname{code}^{15}$. Les deux hommes sont vêtus en rose et bleu et ils demandent à leurs invités de faire de même. Le mariage de Jeanne et Bénédicte est "très protocolaire", avec un long discours de l'adjointe au maire. La dimension festive est également soulignée. Charles et Bertrand, couple très bourgeois, évoquent "une foule de parisiens en costume bleu marine".

Dans les propos de mes enquêté-e-s, la question d'une mise en forme rituelle religieuse semble s'être imposée dans leur trajectoire conjugale dans la foulée du mariage civil pour certain-e-s, avant même le mariage civil pour d'autres (à une époque où il ne leur était pas encore accessible). La référence religieuse surgit à un moment ou un autre de leurs propos. Ainsi, Charles raconte sourire aux lèvres:

\footnotetext{
${ }^{15}$ Le terme "Manif pour tous" désigne tout à la fois les manifestations des opposants au mariage pour tous et le collectif qui en a été l'instigateur et l'organisateur. Les sweatshirts, mais aussi les ballons, fanions, drapeaux et banderoles brandis par les manifestants, étaient rose et bleu, couleurs des layettes que l'on donne traditionnellement pour la première aux petites filles, pour la seconde aux petits garçons. II s'agissait ainsi de marquer symboliquement l'attachement de ces militants anti-gender à la différenciation des sexes, qu'ils jugeaient menacée par l'ouverture du mariage et de l'adoption aux couples gays et lesbiens.
} 
On est sorti de la mairie à midi pile donc l'angélus automatique nous a carillonnés et pleins de copains qui étaient là on dit: ouais! Un mariage carillonné machin! Même l'église est d'accord, regarde! (...). D'ailleurs, il y a une photo de nous avec l'église du village en arrière plan... (Charles, 54 ans, juriste, marié, en couple depuis 10 ans).

Bénédicte et Jeanne se voient proposer par un ami pasteur de bénir leur union. François n'est pas encore marié lorsque je le rencontre (la date a cependant déjà été choisie). L'empressement du jeune homme est certainement atténué par le fait qu'il se considère comme étant "d'une certaine façon déjà marié" du fait de la messe d'action de grâce qui a été dite pour son couple.

Tous et toutes se situent dans le bricolage rituel (Coulmont, 2003) et en sont pleinement conscients. Ainsi, Charles parle de bénédiction puis de messe d'action de grâce. Il semble encore hésiter entre les deux formules. Nicolas décrit le prêtre qui a fait la cérémonie autour de son couple en disant "qu'il improvisait, enfin qu'il composait...". Charles et Bertrand n'ont pas de référence à partir de laquelle ils pourraient penser leur rituel car ils n'ont jamais assisté à "une cérémonie religieuse autour d'un couple d'hommes ou de femmes". En réaction à la mobilisation de la Manif pour tous, Charles entend seulement être béni par "un prêtre haut placé pressenti pour devenir évêque".

\subsection{De discrets bricolages religieux}

Tout en mettant en avant des normes présentées comme universelles et immuables, l'Eglise catholique abrite ainsi en son sein des bricolages et accommodements qui lui permettent de répondre, dans la plus grande discrétion, aux demandes d'accompagnement voire de mise en forme rituelle qui émanent de fidèles vivant en couple de même sexe. Les bricolages réalisés en ces occasions constituent un emprunt plus ou moins formalisé au rituel catholique. Les récits de mes enquêtés permettent d'en distinguer plusieurs types.

La célébration religieuse de l'union de François et Cédric (comme celle projetée par Charles et Bertrand) se présente comme la plus intégrée au dispositif rituel catholique. Les deux hommes sont en couple depuis qu'ils sont étudiants. Ils se sont pacsés à la fin des années 2000. Cette première démarche civile les conduit, quelques mois plus tard, à souhaiter "organiser un temps de prière et d'action de grâce pour ce que 
l'on avait déjà vécu et pour ce que l'on allait vivre ensemble". Comme n'importe quel autre couple catholique, ils s'adressent alors au curé de leur paroisse qui accepte la démarche, une première pour ce prêtre semble-t-il. François et Cédric font une retraite dans une communauté religieuse pour préparer ce moment. Ils y sont conseillés par des prêtres. Au final, 150 personnes, membres de leurs familles (leurs parents sont tous catholiques pratiquants) et amis (dont un bon nombre de catholiques), se trouvent réunies dans l'église paroissiale autour des deux hommes.

On a lu des textes, on a beaucoup chanté. On aime bien Taizé, donc on a pris beaucoup de chants de Taizé. Le prêtre a fait une homélie sur le texte qu'on avait choisi, qui était axé sur la fécondité. Après, on a lu une petite prière à deux, voilà... Nos parents ont apporté des bougies sur l'autel, nos quatre parents, ils sont venus du fond de l'église pour apporter quatre bougies sur l'autel... (...). Cela a vraiment été perçu très positivement et sur tout l'échiquier de nos amis et parents. II y avait une amie juive qui a dit: 'J'ai jamais pleuré comme ça à un mariage'. On a dit: 'C'est pas un mariage'. Elle a repris: 'Oui, à votre pas-mariage, à votre truc-là' [rires]. On avait des amis, donc beaucoup d'amis catholiques qui étaient très émus et très heureux. On avait des gens de nos familles qui venaient... du moins y'en a un qui venait avec les pieds de plomb, hein, enfin qui venait à reculons, tiré par la culotte par sa femme qui lui a dit: 'Ecoute si tu viens pas... ça fait un drame familial donc tu viens, voilà'. Et, à la fin, il est venu nous voir en disant 'ben... merci... je... voilà', et puis il avait pas trop de mots, mais comme on savait qu'il était venu à reculons... Il y avait nos parrains, marraines... Je crois que tout le monde a été assez touché. Après, ce temps de prière on a fait une grande fête avec tout le monde, sur une péniche avec un discours des parents, des amis, un PowerPoint, etc. (François, 34 ans, ingénieur, en couple de même sexe depuis 15 ans).

Nicolas aspire lui aussi, dans la foulée de son Pacs avec Louis à la fin des années 2000, à une mise en forme rituelle de son union. C'est sa mère, elle-même catholique et très investie dans l'institution ecclésiale, qui le met en contact avec un prêtre, proche du mouvement David et Jonathan ${ }^{16}$. Avec lui, ils se lancent dans une préparation au mariage qui

\footnotetext{
${ }^{16}$ Ce mouvement, fondé à Paris en 1970, regroupe des homosexuel-le-s catholiques. Leur militantisme est discret et demeure aux marges de l'institution ecclésiale. Les revendications portées publiquement ne sont pas très subversives, notamment si on la compare au militantisme chrétien gay nord-américain. Ainsi, si David et Jonathan ont pris
} 
les conduit à faire une retraite dans un monastère. En revanche, le rituel qu'ils inventent à cette occasion (un échange dialogué avec un prêtre sous un arbre, en compagnie d'une centaine d'invité-e-s), ne s'apparente pas directement à une prière du rituel catholique et n'a pas lieu dans une église.

Jeanne, de son côté, décrit le baptême de leur fille, comme un moment de réaffiliation à l'Eglise et de réintégration à sa structure paroissiale:

Avec le baptême de Madeleine, on a vraiment eu l'impression qu'on se réintégrait pleinement à l'Eglise. On n'a plus les craintes qu'on avait avant. On s'assume. On veut l'éveiller à la foi. On ne craint plus d'aller toutes les deux en paroisse, de s'y impliquer ensemble. (Jeanne, 40 ans, profession libérale, mariée, en couple depuis 20 ans).

Le prêtre de plus de 80 ans, qui baptiste l'enfant, n'appartient pas à un réseau d'Eglise sensibilisé aux questions LGBT. C'est un vieil ami de la famille de Bénédicte. Il s'en est d'ailleurs ouvert, un peu par hasard, à son évêque en visite dans le coin la veille du baptême, évêque très mobilisé contre le mariage pour tous. Au cours de la cérémonie, elles présentent toutes les deux l'enfant en tant que parents. Le seul problème semble être celui des registres où la configuration de deux mères n'est pas prévue:

- La question c'était sur le registre. Mais pour le baptême c'était comme un... y'avait pas de problème du tout.

-Et alors comment a-t-elle été résolue cette histoire de registre?

一: Je ne sais plus, il n'a pas barré père... [Rires]

-B: Je suis témoin je crois. Et j'ai signé le registre en tant que témoin.

-J: Oui mais il a barré témoin, il a mis autre chose et...

-B: Je ne sais plus, enfin il a bidouillé.

(Bénédicte et Jeanne, 40 ans, la première est chercheuse et la seconde exerce une profession libérale, mariées, en couple depuis 20 ans).

La plasticité du rituel du baptême avait déjà été bien mise en évidence par Martine Gross: "La naissance spirituelle qu'est le baptême est dans le contexte homoparental, une naissance sociale, l'avènement public

officiellement position en faveur du mariage pour tous, il ne porte pas de demande de mariage religieux pour les couples de même sexe. Une réflexion a été récemment ouverte sur la possibilité d'une bénédiction de ces couples. 
d'une famille" (2004: 187). On peut même dire qu'il end possible une reconnaissance du couple de même sexe, en tant que parents. C'est ce que relatent Jeanne et Bénédicte, mariées depuis l'été 2013. C'est le baptême de leur fille qui a constitué à leurs yeux l'occasion d'une première reconnaissance ecclésiale de leur couple.

Ces pratiques de mise en forme rituelle des unions de même sexe et de baptême en contexte homoparental avec une pleine reconnaissance des deux parents sont connues en interne, au sein du clergé, y compris des évêques même les plus conservateurs. De ce point de vue, on ne peut pas les qualifier de clandestines. En revanche, elles doivent rester suffisamment discrètes pour ne pas exposer publiquement les bricolages avec la règle. Elles ne s'accompagnent d'aucune revendication portée publiquement. Le principe est celui de non publicité:

Lors de la retraite au monastère, on se retrouve tous les trois avec le frère hôtelier à l'arrivée, qui fait partie de l'accueil. Ils se connaissent avec le prêtre qui est déjà venu, ils se sont déjà vus. Vient la question, à laquelle moi je ne m'attendais pas: 'Pourquoi vous venez là?', demande le frère. Je réponds... je sais plus quoi exactement, mais en gros qu'on se prépare à un engagement. Le truc était neutre et en même temps clair, enfin neutre... Le prêtre me dira juste après: 'Tu as très bien répondu'. Cela voulait dire aussi qu'il avait peut-être un peu flippé. J'ai compris qu'il fallait, tout en disant les choses, ne pas trop en dire quand même... Pas être militant, on va dire. (Nicolas, 41 ans, enseignant, marié, en couple depuis 7 ans).

C'était un moment assez extraordinaire, sur lequel on a essayé d'être plutôt discrets pour pas mettre en difficulté les deux prêtres qui ont accepté de nous accompagner. (François, 34 ans, ingénieur, en couple de même sexe depuis 15 ans, à propos du rituel posé dans sa paroisse).

Il faut savoir jouer sur les mots. Ainsi, autre principe, le terme "mariage" doit être soigneusement évité (comme le demande explicitement le curé qui répond à la demande de François et Cédric), le mariage religieux restant dans le catholicisme le monopole des couples hétérosexuels. Ainsi, Charles et Bertrand envisagent une messe d'action de grâce. L'ami prêtre auquel ils se sont adressés a souhaité que ce moment rituel soit "déconnecté" du mariage civil. "On ne fait pas la mairie, l'église, la réception", souligne Charles qui n'entend pas "singer le mariage": 
On ne va pas se mettre au milieu de l'église sur les fauteuils, on ne va pas demander une bénédiction spécifique, on ne va pas demander que de nombreux amis nous entourent et que notre foyer accueille de nombreux enfants, mais on va simplement dire ce qu'on est... devant des gens qui ont envie de l'entendre. Voilà. (...). On va inviter cent personnes dans une église quelque part... avec un petit buffet à la sortie ou un diner assis, enfin on verra bien, mais... en leur disant, comme invitation dix ans de vie commune, voilà, on vient les fêter sous le regard de Dieu... Comme un anniversaire de mariage. (Charles, 54 ans, juriste, marié, en couple depuis 10 ans).

Le couple que forment ces deux hommes a d'ailleurs déjà été béni, dans un cadre privé, à l'occasion de leur installation dans la maison de campagne qu'ils ont acquis ensemble:

Quand on s'est installé en Normandie, on a demandé à un ami prêtre de bénir la maison. Il a trouvé une formule, il a béni la maison et il a dit: 'Ah ben de toute façon comme vous êtes là vous pouvez vous tenir la main, enfin en gros, vous êtes bénis aussi, donc je bénis votre couple par la même occasion'. Bon, voilà. C'est un prêtre qui est un ami. (Bertrand, 45 ans, courtier en assurance, marié, en couple depuis 10 ans).

\subsection{Des héritier-ère-s}

Les disparités et les inégalités sociales dans le recours aux cérémonies de mariages et aux formes qui lui sont données ont été mises en évidence à propos des unions de sexe différents (Bozon, 1992; Maillochon, 2014). Florence Maillochon met ainsi au jour "la diversité sociale des pratiques de mariage et les profondes inégalités sur lesquelles elles reposent au-delà de l'apparente uniformisation de la fête" (2014: 140). Ces dernières s'expliquent par la disparité des ressources économiques, relationnelles et sociales des conjoints. Si la célébration personnalisée du couple est devenue la norme de la fête de mariage, elle "demeure cependant inégalement orchestrée pour deux raisons intimement liées, tenant, d'une part à l'origine et au parcours social des époux, et, d'autre part, à la quantité et à la qualité de leurs invités" (Ibid.: 150). La tendance à la personnalisation ne vaut pas seulement pour la fête qui suit l'union. Elle vaut tout autant pour la cérémonie religieuse, lorsque les couples y ont recours. Il s'agit, comme dans le cas des funérailles, non "pas de reconduire une forme instituée, mais de concevoir sur mesure (...) quelque chose d'unique et d'authentique" (Déchaux, 2001: 172-173). Le souhait de personnalisation 
peut se manifester par la préférence pour des textes ou des chants profanes. Les demandes vont parfois assez loin, jusqu'à l'euphémisation du caractère religieux de la cérémonie. Le degré de personnalisation atteint au final dépend de la négociation qui a eu lieu, permettant un ajustement entre les demandes des couples et les exigences de l'institution ecclésiale. On peut estimer qu'il est d'autant plus grand que “les intéressés, dotés d'un capital culturel élevé et d'une capacité rhétorique supérieure à celle du prêtre, sont parfaitement capables de lui administrer la démonstration de la pertinence de leur choix" (Hervieu-Léger, 2003: 323).

Mes enquêté-e-s mettent à profit leurs ressources non pas pour négocier une moindre place du religieux mais au contraire pour obtenir d'un prêtre une mise en forme rituelle, qui sans avoir de valeur sacramentelle emprunte au répertoire catholique, alors même que leur état de vie (leur conjugalité et leur parentalité) est officiellement désapprouvé par l'Eglise.

Tous et toutes ont grandi dans une milieu bourgeois, ont effectué des études supérieures (plusieurs sont passés par une grande école) et exercent aujourd'hui une profession qui les rattache à la catégorie socioprofessionnelle des "cadres et professions intellectuelles supérieures". Mes enquêté-e-s apparaissent comme des héritiers, au sens qu'en donne la sociologie bourdieusienne (Bourdieu et Passeron, 1964), mais aussi dans une perspective religieuse. C'est ainsi qu'ils se présentent. Au sein de leur fratrie pourtant nombreuse (au moins trois enfants, souvent quatre, jusqu'à sept), plusieurs ont insisté sur le fait que c'est à eux et non à leurs frères et sœurs que leurs parents ont le mieux transmis leurs croyances, pratiques et valeurs religieuses.

Je suis le seul qui va encore à la messe. C'est un peu drôle, je suis marié... les autres ne sont pas mariés à l'église. J'ai un frère qui est divorcé et a une nouvelle compagne... (Nicolas, 41 ans, enseignant, marié, en couple depuis 7 ans).

Ils et elles sont issus de familles nombreuses, pratiquantes, très intégrées au catholicisme. Ils ont reçu une socialisation primaire et secondaire au catholicisme. Ils ont fréquenté le catéchisme, l'aumônerie et très souvent le scoutisme. Le plus âgé a été enfant de chœur. Beaucoup ont fait leur scolarité dans l'enseignement privé confessionnel. Plusieurs des hommes de mon échantillon se sont posés la question de la vocation religieuse, ce qui n'a rien d'étonnant l'attrait 
pour le célibat consacré ayant longtemps constitué et constituant certainement encore aujourd'hui un moyen légitime d'échapper au mariage hétérosexuel dans ces milieux conservateurs. Aujourd'hui encore, ils ont des engagements multiples dans l'Eglise, en paroisse mais également dans les mouvements et associations (le Secours Catholique, par exemple).

Ajoutons que l'homogamie religieuse est très fréquente chez ces couples. François et Cédric se sont rencontrés à l'aumônerie de la grande école où ils ont été étudiants. Jeanne et Bénédicte étaient cheffes scoutes et ont fait connaissance à l'occasion d'une formation proposée par le mouvement. Si Bertrand n'était pas catholique, il s'est converti après sa rencontre avec Charles.

Ce sont des catholiques bien dotés en capitaux en tous genre, en capital culturel de type théologique, qui leur permet de puiser dans le large répertoire de rituels catholiques, et en capital social ecclésial qui rend possible la mobilisation de prêtres dans leurs réseaux catholiques. Ils savent parfaitement ce qu'ils peuvent et ne peuvent pas faire. Ils savent aussi comment et à qui formuler leur demande de rituel.

\section{CONCLUSION}

On le voit, malgré un discours officiel de désapprobation des unions de même sexe qui s'est trouvé réaffirmé avec force en 2012-2013, I'Eglise catholique a répondu positivement à des demandes de mise en forme rituelle émanant de fidèles homosexuels. La mobilisation contre le mariage pour tous n'a pas changé la donne: certains rituels ont été posés avant (dans la foulée d'un Pacs), d'autres après.

L'échantillon que forme mes enquêté-e-s, couples d'hommes et de femmes très pratiquant-e-s et très attachées à l'Eglise catholique, est cependant très spécifique. Obtenir un accompagnement voire une mise en forme rituelle d'une union de même sexe par un prêtre catholique nécessite un important capital culturel de type théologique et un large capital social ecclésial. Ce n'est pas à la portée de tous les couples. II s'agit là d'une hypothèse que la poursuite de la recherche, par une diversification de l'échantillon, devra confirmée mais qui paraît assez solidement fondée. Ajoutons que la demande de mariage n'est pas partagée par l'ensemble des gays et lesbiennes catholiques. Le matériau recueilli n'est donc nullement représentatif. Certain-e-s, catholiques ou 
non, ont d'ailleurs pu s'opposer à la loi ouvrant le mariage au couple de même sexe et, ainsi, "militer contre leurs droits" (Durand, 2015).

Par-delà l'homophobie qui s'est exprimée, l'épisode du mariage pour tous, une fois la loi promulguée et la mobilisation retombée, a aussi eu un effet inattendu dans le monde catholique, celui d'un début de banalisation des couples de même sexe et des familles homoparentales. On peut ainsi noter des initiatives pastorales diocésaines en faveur d'un meilleur accueil ecclésial des personnes homosexuelles. Cet effet s'est trouvé conjugué à ceux du premier synode romain sur la famille convoqué par le pape François à l'automne 2014. C'est dans ce contexte qu'une prise de parole tout à fait inédite dans le catholicisme français a eu lieu, celle d'un prêtre du diocèse de Paris, qui s'est prononcé en faveur de la recherche "d'une forme de reconnaissance d'un amour fidèle et durable entre deux personnes de même sexe, quel que soit leur degré de chasteté"17 $^{17}$, cette reconnaissance pouvant selon lui prendre d'un point de vue liturgique la forme d'une prière (dans un premier temps, il a même été question d'une bénédiction mais le prêtre est revenu quelques jours plus tard sur une telle éventualité).

\section{BiBLIOGRAFÍA}

Bellamy, V. \& Beaumel, C. (2014). Bilan démographique 2013. Trois mariages pour deux Pacs. Paris: Insee Première, $n^{\circ} 1482$.

Béraud, C. (2014). Un front commun des religions contre le mariage pour tous ? Contemporary French Civilization, 39(3), 335-349.

Béraud, C. (2015). Des catholiques favorables au mariage pour tous ? Retour sur la mobilisation de 2012-2013 en France. Sextant. Revue de la structure de recherche interdisciplinaire sur le genre, l'égalité et la sexualité (STRIGES), 31, 131-144. Dossier "Habemus Gender! Déconstruction d'une riposte religieuse " coordonnée par D. Paternotte, S. Van der Dussen \& V. Piette.

Béraud, C. \& Portier, P. (2015). Métamorphoses catholiques. Acteurs, enjeux et mobilisations depuis le mariage pour tous. Paris: Editions de la $\mathrm{MSH}$.

Besson, C. (2012). Homosexuels catholiques. Sortir de l'impasse. Paris: Ed. de l'Atelier.

\footnotetext{
${ }^{17}$ La Croix, 7 octobre 2014. 
Bourdieu, P. \& Passeron, J.-C. (1964). Les héritiers : les étudiants et la culture. Paris: Editions de Minuit.

Borillo, D. \& Lascoumes, P. (2002). Amours égales : le Pacs, les homosexuels et la gauche. Paris: Ed. La Découverte.

Bozon, M. (1992). Sociologie du rituel de mariage. Population, 2, 409-434.

Buisson-Fenet, H. (2004). Un sexe problématique. L'Eglise et l'homosexualité en France 1971-2000. Saint-Denis: Presses Universitaire de Vincennes.

Chateauraynaud, F. \& Torny, D. (1999). Les sombres précurseurs : une sociologie pragmatique de l'alerte et du risque. Paris: Editions de I'EHESS.

Clanché, P. (2014). Mariage pour tous. Divorce chez les cathos. Paris: Plon.

Congrégation de la doctrine de la foi (2003). Considérations à propos des projets de reconnaissance juridique des unions entre personnes homosexuelles.

Disponible

en:

http://www.vatican.va/roman_curia/congregations/cfaith/documen ts/rc_con_cfaith_doc_20030731_homosexual-unions_fr.html. Última consulta: 31/07/2016.

Coulmont, B. (2003). "Que Dieu vous bénisse ! " : le mariage religieux des couples du même sexe aux Etats-Unis. Tesis doctoral en Sociología. Paris: EHESS.

Déchaux, J.-C. (2001). La mort dans les sociétés modernes : la thèse de Norbert Elias à l'épreuve. L'Année sociologique, 51, 161-183.

Déloye, Y. (2006). Les voix de Dieu. Pour une autre histoire du suffrage électoral : le clergé catholique français et le vote : XIXe - XXe siècle. Paris: Fayard.

Dobbelaere, K. \& Pérez-Agote, A. (Eds.) (2015). The Intimate. Polity and the Catholic Church. Leuven: KADOC Studies on Religion, Culture and Society, Leuven University Press.

Durand, M. (2015). Militer contre ses droits. Les mobilisations d'homosexuel-le-s anti-Mariage pour tous. Congrès national de I'Association française de science politique, session thématique "Mobilisations conservatrices autour d'enjeux sexuels et reproductifs", Aix-en-Provence.

Fassin, E. (2001). La voix de l'expertise et les silences de la science dans le débat démocratique. In E. Fassin \& D. Borrillo (Eds.), Au-delà du Pacs : l'expertise familiale à l'épreuve de l'homosexualité (pp. 89-110). Paris: PUF. 
Gross, M. (2004). Baptêmes catholiques en contexte homoparental. In E. Dianteill, D. Hervieu-Léger \& I. Saint-Martin (Eds.), La modernité rituelle. Rites politiques et religieux des sociétés modernes (pp. 179194). Paris: L'Harmattan.

Gross, M. (2008). Etre chrétien et homosexuel en France. Sociétés contemporaines, 71, 67-93.

Gross, M. (2012). De la honte à la revendication identitaire : être catholique et homosexuel en France. In C. Béraud, F. Gugelot \& I. Saint-Martin (Eds.), Catholicisme en tensions (pp. 215-227). Paris: Éditions de I'EHESS.

Hervieu-Léger, D. (2003). Catholicisme, la fin d'un monde. Paris: Bayard.

Maillochon, F. (2014). La fête du mariage, un marqueur des inégalités sociales en France. In S. Paugam (Ed.), L'intégration sociale. Force, fragilité et rupture des liens sociaux (pp. 139-153). Paris: PUF.

Maillochon, F. (2016). La passion du mariage. Paris: PUF.

Paternotte, D. (2011). Revendiquer le " mariage gay ". Belgique, France, Espagne. Bruxelles: Éditions de l'Université de Bruxelles.

Portier, P., Théry, T., Béraud, C. \& Coulmont, C. (2015). Du mariage civil au "mariage pour tous". Sécularisation du droit et mobilisations catholiques. Sociologie, 6(1), 81-104.

Pichardo Galàn, J.-I. (2008). Le mariage homosexuel au pays de la famille. In V. Descoutures, M. Digoix, E. Fassin \& W. Rault (Eds.), Mariages et homosexualités dans le monde (pp. 62-71). Paris: Autrement.

Pullman, B. (2010). Mille et une façons de faire des enfants. La révolution des méthodes de contraception. Paris: Calmann-Lévy.

Rault, W. (2007). Nouvelles formes d'union, nouveaux rituels. Le Pacs comme expression d'un engagement privé ? Informations sociales, 144, 86-94.

Rault, W. (2008). Ce que le Pacs fait au mariage gai et lesbien. In V. Descoutures (Ed.), Mariages et homosexualités dans le monde (pp. 117-125). Paris: Autrement.

Rault, W. (2016). Les attitudes gayfriendly en France : entre appartenances sociales, trajectoires familiales et biographies sexuelles. Actes de la recherche en sciences sociales, 213, 38-65.

Rault, W. \& Letrait, M. (2009). Choix du pacte civil de solidarité et rapport à la religion. Politiques sociales et familiales, 96, 41-54.

Segalen, M. (2003). Eloge du mariage. Paris: Découvertes Gallimard. 